\title{
Ruído no lazer: uso de estéreos pessoais com fones de ouvido durante atividades físicas
}

\author{
Noise at leisure: use of personal stereos with headphones during physical activities
}

Ruido en el tiempo libre: uso de equipos de música personales con auriculares durante las actividades físicas

\section{Pollyana Nascimento DIEDIO}

Mariane AMARAL ${ }^{2}$

Juliana DE CONTO

${ }^{1}$ Departamento de Fonoaudiologia, Universidade Estadual do Centro-Oeste, UNICENTRO, Campus Irati, 84505-677 Irati- PR, Brasil

${ }^{2}$ Professora colaboradora no curso de Fonoaudiologia da Universidade Estadual do Centro Oeste (UNICENTRO) na área de Audiologia. Doutoranda no Programa de Pós-Graduação Interdisciplinar em Desenvolvimento Comunitário da UNICENTRO. Mestre em Desenvolvimento Comunitário (2018) e graduada em Fonoaudiologia (2014) pela UNICENTRO

${ }^{3}$ Pós-doutorado em Distúrbios da Comunicação. Doutora e Mestre em Engenharia de Produção na área de Ergonomia pela Universidade Federal de Santa Catarina (2009), Especializada em Audiologia Clínica, graduada em Fonoaudiologia pela Universidade do Vale do Itajaí (1996) e Ciências Matemáticas pela Universidade Estadual do Oeste do Paraná (1988). Atua na área de Fonoaudiologia, com ênfase em Audiologia. Pesquisa de interesse: Ergonomia, Gestão da Informação em Saúde Coletiva, Fonoaudiologia, Audiologia Clínica, Audiologia aplicada à Saúde do Trabalhador, Controle de Ruído, Ruído Ambiental, Docente da UNICENTRO, Campus Irati, 84505-677 Irati- PR, Brasil

\section{Resumo}

Introdução: Academias de ginástica utilizam músicas em intensidade elevada como forma de motivação para as atividades físicas, apesar disto muitos jovens optam por utilizar simultaneamente, fones de ouvido de seus estéreos pessoais. Objetivo: Investigar os efeitos auditivos e extra-auditivos causados pela música amplificada por estéreos pessoais com fones de ouvido durante atividades físicas. Metodologia: Participaram do estudo 31 adultos frequentadores de academias, usuários de estéreos pessoais. Foi aplicado questionário para investigação de efeitos auditivos e extra auditivos e realizada medição do nível de ruído ambiente da academia e dos estéreos pessoais de cada participante. Resultados: A maioria dos participantes utiliza fones de ouvido de uma a duas horas diárias nas academias, com maior frequência de cinco a seis dias por semana. Alguns participantes apresentam no cotidiano sintomas de redução da audição e plenitude auricular, bem como irritação e dor de cabeça. Os efeitos auditivos mais relatados após o uso de estéreos pessoais foram a plenitude auricular, redução da audição e zumbido, enquanto os efeitos extra auditivos mais relatados foram a dor de cabeça e a insônia. Observou-se que o NPS dos estéreos pessoais, usados simultaneamente ao som da academia, teve média de 95,4 dBNPS e quanto ao ruído ambiente das academias, a média foi de 78,1 dBNPS. Conclusão: As queixas auditivas e extra auditivas relatadas após o uso de fones de ouvido, continuaram sendo as mesmas do cotidiano, como plenitude auricular, dor de cabeça e insônia, mas com maior incidência.

Descritores: Audição; Fonoaudiologia; Música; Ruído.

\section{Abstract}

Introduction: Gymnasiums use loud music as a form of motivation for physical activities, despite this, many young people choose to use headphones of their personal stereos simultaneously. Objective: To investigate auditory and extra-auditory effects caused by music amplified by personal studios with headphones during activities. Methodology: Participated in the study 31 adults attending gym, users of personal stereos. A questionnaire was applied to investigate auditory and extra-auditory effects and carried out at the level of ambient noise in the gym and the personal stereos of each participant. Results: Most participants use headphones for one to two hours at the gyms, with a higher frequency of five to six days a week. Some participants experience daily symptoms of reduced hearing and ear fullness, as well as irritation and headache. The most related auditory effects after using personal stereos were ear fullness, reduced hearing and tinnitus, while the most related extra auditory effects were headache and insomnia. Note that the NPS of personal stereos, used simultaneously with the sound of the gym, had an average of $95.4 \mathrm{dBNPS}$ and for the sound environment of the gyms, with an average of $78.1 \mathrm{dBNPS}$. Conclusion: As related auditory and extra auditory queues after using headphones, they remain the same as in everyday life, such as ear fullness, headache and insomnia, but with a higher incidence.

Descriptors: Hearing; Speech Therapy; Music; Noise.

\section{Resumen}

Introducción: Gimnasios usan música a todo volumen como una forma de motivación para las actividades físicas, a pesar de esto, muchos jóvenes eligen usar los auriculares de sus equipos de música personales simultáneamente. Objetivo: investigar los efectos auditivos y extraauditivos causados por la música amplificada por estudios personales con auriculares durante las actividades. Metodología: Participaron en el estudio 31 adultos que asistieron al gimnasio, usuarios de equipos de música personales. Se aplicó un cuestionario para investigar los efectos auditivos y extraauditivos y se realizó al nivel del ruido ambiental en el gimnasio y los equipos de música personales de cada participante. Resultados: la mayoría de los participantes usan auriculares durante una o dos horas en los gimnasios, con una frecuencia más alta de cinco a seis días a la semana. Algunos participantes experimentan síntomas diarios de disminución de la audición y la plenitud del oído, así como irritación y dolor de cabeza. Los efectos auditivos más relacionados después de usar equipos de sonido personales fueron la plenitud del oído, la audición reducida y el tinnitus, mientras que los efectos auditivos adicionales más relacionados fueron dolor de cabeza e insomnio. Tenga en cuenta que el NPS de los equipos de sonido personales, utilizados simultáneamente con el sonido del gimnasio, tenía un promedio de 95.4 dBNPS y para el entorno de sonido de los gimnasios, con un promedio de 78.1 dBNPS. Conclusión: como las colas auditivas y extraauditivas relacionadas después de usar auriculares, siguen siendo las mismas que en la vida cotidiana, como la plenitud del oído, dolor de cabeza e insomnio, pero con una mayor incidencia.

Descriptores: Audiencia; Terapia del Habla; Musica; Ruido.

INTRODUÇÃO

A música é vista como um som agradável, geralmente associada a fatos importantes da vida de cada pessoa, pois proporciona prazer a quem ouve, e, portanto, é vista como sendo incapaz de causar algum dano ao ser humano'.

Dentro das academias de ginástica, a música é indispensável, pois colabora para tornar o ambiente mais agradável e criar uma atmosfera que, no campo subjetivo, é eficaz no sentido de conforto e bem-estar, ao compreender que, quanto mais elevada a intensidade da música, maior o estímulo às atividades físicas ${ }^{2}$. A música pode aumentar a 
motivação, ajudando na distração das dores musculares e ainda provocar alterações em respostas fisiológicas, como na frequência cardíaca $^{3}$. Além disso, com a música motivando e distraindo o atleta em suas atividades físicas, faz com que ele se exercite por maior tempo, mascarando o efeito da exaustão ${ }^{3}$. A música também promove 0 relaxamento durante 0 exercício, melhorando assim a performance do atleta ${ }^{4}$.

Ainda, as atividades físicas podem ser consideradas como uma forma de lazer quando ocorrem nos períodos de tempo disponíveis entre as obrigações sociais ou, também, quando relacionadas a atividades em ginásios, esportes, passeios, etc ${ }^{5}$. Atualmente, já é fato conhecido que as pessoas procuram as academias em busca da forma física e do bem-estar sendo, portanto, uma forma de lazer ${ }^{2}$.

Sendo assim, a música além de motivar durante o exercício, também é aliada de uma melhor performance nas atividades físicas. Contudo, é necessário levar em consideração que ouvir música em intensidade elevada no trabalho ou no lazer, durante atividades físicas, pode acarretar em prejuízos irreversíveis para a audição e dificultar a comunicação do indivíduo.

Em academias, além da música ambiente em intensidade elevada, muitos frequentadores utilizam simultaneamente equipamentos estéreos pessoais com fones de ouvido. Nos últimos anos, o uso de estéreos pessoais para ouvir música aumentou e se tornou um hábito de muitos jovens ao utilizá-lo em atividades físicas como caminhadas, passeios de bicicleta e em academias ${ }^{6}$.

Atualmente, 0 equipamento estéreo pessoal é acoplado em celulares e MP3 players, possibilitando que a música seja levada a todos os lugares, e, além de ser de fácil uso, tornouse um acessório quase indispensável para muitas pessoas. Tais aparelhos permitem aos usuários ouvir música sem interrupção por períodos prolongados de tempo e em níveis sonoros que podem representar risco à audição ${ }^{7}$, pois a maioria dos equipamentos de som portáteis pode atingir facilmente os 130 decibéis $(\mathrm{dB})^{8}$.

Frequentemente, os fones de ouvido de tais aparelhos são utilizados em intensidades elevadas de forma que, as pessoas ao redor, são capazes de ouvir claramente o som que está sendo reproduzido na orelha do indivíduo com o dispositivo ${ }^{9}$.

A exposição a sons intensos, seja ruído ocupacional ou no lazer, pode resultar em perda auditiva induzida pelo ruído (PAIR $)^{9}$. No caso da exposição contínua e intensa à música, podemos nomear de perda auditiva induzida pela música (PAIM). Este tipo de perda auditiva apresenta instalação lenta e gradual, de forma que as pessoas não notam que estão sendo expostas a um risco ${ }^{9}$, que só é percebido quando atinge um grau acentuado, afetando a comunicação humana de forma irreversível ${ }^{10}$.

Além da perda auditiva, a PAIM e a PAIR podem apresentar outros sintomas auditivos, entre os quais estão o zumbido, a dificuldade de discriminação dos sons e a plenitude auricular e ainda sintomas extras-auditivos, como mudanças comportamentais, alterações cardiovasculares, vestibulares, digestivas e neurológicas ${ }^{11}$.

Diante do exposto, o objetivo deste trabalho é investigar os efeitos auditivos e extra auditivos do uso de estéreos pessoais com fones de ouvido durante atividades físicas bem como, verificar o nível de pressão sonora dos estéreos pessoais dos participantes e o ruído ambiental das academias.

MATERIAL E MÉTODO

Trata-se de um estudo descritivo com análise de dados de caráter quantitativa, realizado após a aprovação do Comitê de Ética em Pesquisa da instituição sob parecer $n^{0}$ 2.813.552. Todos os participantes assinaram o Termo de Consentimento Livre e Esclarecido e todas as academias autorizaram a realização da pesquisa, ambos tendo suas identidades preservadas, sendo garantido sigilo absoluto em relação aos dados pessoais dos indivíduos e estabelecimentos.

Participaram do presente estudo adultos jovens que utilizam estéreos pessoais com fones de ouvido durante a realização de atividades físicas, em quatro academias de ginástica localizadas em uma cidade na região sudeste do estado do Paraná. As academias serão apresentadas no texto da seguinte forma: Academia 1, Academia 2, Academia 3 e Academia 4. Primeiramente os participantes, selecionados aleatoriamente, responderam a um questionário elaborado pelas pesquisadoras para caracterização da amostra e para coleta de dados de saúde geral e saúde auditiva, bem como dados referentes ao tipo e frequência do uso de estéreos pessoais com fones de ouvido, gosto musical e presença de desconfortos auditivos e extra auditivos após o uso dos dispositivos. Após a aplicação do questionário, foi realizada a medição do nível do ruído ambiente de cada academia, no qual era anotado o valor de dez medições do ruído ambiente e então realizada a média desses valores para achar o nível de ruído de cada academia. Também foi realizada a medição do 
nível de pressão sonora do estéreo pessoal de cada participante, sendo instalado um tubo de plástico, no qual uma das pontas estava conectada no microfone do decibelímetro e na outra ponta era acoplado o fone de ouvido de cada participante com a música e a intensidade que estava ouvindo no momento que estava se exercitando. Nos dois processos foi utilizado o decibelímetro Digital Sound Level Meter, da marca Benetech, modelo GM1351 (Shenzhen, Guangdong, China) e a medição foi realizada em dB nível de pressão sonora (NPS).

Como ação final, foi elaborado um cartaz (Figura 1) de conscientização para colocar nas academias participantes, como uma forma de alerta sobre o uso de estéreos pessoais e as consequências que podem acarretar para a audição.

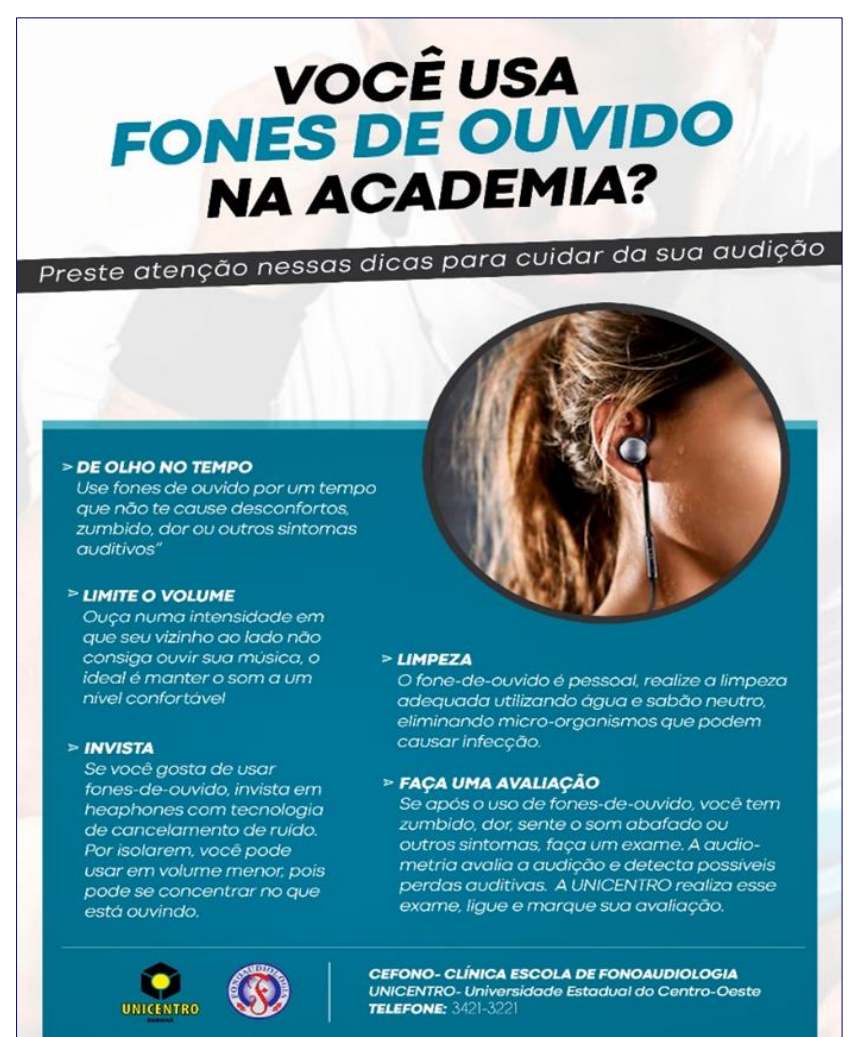

Figura 1: Cartaz de conscientização sobre o uso de estéreos pessoais.

\section{RESULTADOS E DISCUSSÃO}

A casuística foi composta por 31 indivíduos, sendo dez participantes do sexo feminino e 21 do sexo masculino, com média de idade de 23,35 anos, variando entre 18 e 34 anos. Informações detalhadas sobre a idade dos participantes, de acordo com sexo e academia frequentada, podem ser vistas na Tabela 1.

Todos os participantes do estudo utilizam os estéreos pessoais com fones de ouvido sempre que estão realizando atividades físicas na academia, permanecendo neste ambiente por volta de uma a duas horas diárias, por ser a variação de tempo de treino. Em relação à frequência semanal nas academias, 22 participantes (71\%) relataram frequentar de cinco a seis dias na semana e nove $(29 \%)$ relataram ir à academia de três a quatro vezes por semana. Semelhante ao encontrado no presente estudo, uma pesquisa realizada com 150 participantes de uma academia que responderam um questionário sobre exercícios e suplementos, mostrou que $54,76 \%$ dos sujeitos relataram praticam atividade com duração entre 60 e 90 minutos diários, de 3 a 5 vezes por semana ${ }^{12}$.

Tabela 1. Idade dos participantes de acordo com o sexo e academia frequentada

\begin{tabular}{|c|c|c|c|c|}
\hline Academia & Idade & $\begin{array}{c}\text { Sexo } \\
\text { feminino }\end{array}$ & $\begin{array}{c}\text { Sexo } \\
\text { masculino }\end{array}$ & Total \\
\hline \multirow{5}{*}{ Academia 1} & $18-20$ anos & 1 & 7 & 8 \\
\hline & $21-23$ anos & 3 & 2 & 5 \\
\hline & $24-26$ anos & 1 & 2 & 3 \\
\hline & $27-29$ anos & 1 & 4 & 5 \\
\hline & $30-34$ anos & 1 & o & 1 \\
\hline \multirow{6}{*}{ Academia 2} & $21-23$ anos & 1 & o & 1 \\
\hline & $24-26$ anos & o & 1 & 1 \\
\hline & $27-29$ anos & O & 1 & 1 \\
\hline & $18-20$ anos & o & 2 & 2 \\
\hline & $27-29$ anos & 1 & 1 & 2 \\
\hline & $30-34$ anos & 1 & o & 1 \\
\hline Academia 4 & $18-20$ anos & O & 1 & 1 \\
\hline
\end{tabular}

Quando questionado aos participantes sobre os motivos que os levaram a utilizar os estéreos pessoais em academias, os relatos vieram no sentido de que a música no estéreo pessoal funciona como uma forma de concentração $(45 \%) \quad(n=14)$, que a música ambiente da academia não agrada (39\%) $(n=12)$, a música é uma forma de distração durante a realização das atividades físicas $(16 \%)(n=5)$, a música é uma forma de estímulo para a realização das atividades físicas (13\%) $(n=4)$ e que a música é uma forma de motivação $(3 \%)(n=1)$, sendo que alguns dos participantes relataram mais de um motivo pelo uso de estéreos pessoais.

Silva e Gress $^{13}$ realizaram estudo com 144 praticantes de exercício físico com frequência regular em academias e notaram dado semelhante ao da presente pesquisa, no qual $15,7 \%$ dos participantes referem a música como uma forma de distração durante as atividades físicas. Contudo, o mesmo estudo mostra que, diferente dos resultados encontrados nesta pesquisa, ouvir música como forma de concentração foi relatada por apenas $9,4 \%$ dos participantes, enquanto a motivação foi referida por $50,3 \%$ dos sujeitos. Outro estudo realizado com um grupo de mulheres praticantes de ginástica de academia também mostrou que a música é o maior fator motivante da aula de ginástica ${ }^{14}$.

Sobre o uso do estéreo pessoal com 
fones de ouvido no cotidiano, 18 participantes $(58 \%)$ relataram fazer seu uso, com maior incidência na parte da tarde $(39 \%) \quad(n=7)$, seguindo pelo período noturno (34\%) $(n=6)$, na parte da manhã $(28 \%)(n=5)$, durante todo o dia $(22 \%) \quad(n=4)$ e antes de dormir $(11 \%) \quad(n=2)$. Alguns participantes relataram utilizar em mais de um período do dia e, além disso, parte considerável dos participantes $(78 \%)(n=24)$, relatam frequentar locais de lazer com música amplificada em média uma vez na semana. Tal dado corrobora com estudo realizado por Gonçalves e $\operatorname{Dias}^{15}$ no qual $66,67 \%$ dos participantes relataram estar expostos a NPS elevados em média uma vez na semana.

Em relação aos aspectos de saúde geral e auditiva dos participantes, a frequência de sintomas relatados como comuns no dia-a-dia pode ser vista detalhadamente na Tabela 2.

Tabela 2. Sintomas auditivos e extra auditivos relatados pelos participantes como frequentes em seu cotidiano

\begin{tabular}{c|c|c}
\hline Sintomas & $\begin{array}{c}\text { Frequência } \\
\text { Absoluta } \\
\text { (n) }\end{array}$ & $\begin{array}{c}\text { Frequência } \\
\text { Relativa } \\
\text { (\%) }\end{array}$ \\
\hline Redução da audição & 9 & $30 \%$ \\
\hline Plenitude Auricular & 7 & $23 \%$ \\
\hline Vertigem & 5 & $16 \%$ \\
\hline Zumbido & 5 & $16 \%$ \\
\hline Otalgia & 1 & $3 \%$ \\
\hline Dor de cabeça & 18 & $60 \%$ \\
\hline Dificuldade de concentração & 14 & $45 \%$ \\
\hline Irritação & 13 & $42 \%$ \\
\hline Alteração de Pressão & 11 & $36 \%$ \\
\hline Insônia & 11 & $36 \%$ \\
\hline Alterações gástricas ou intestinais & 8 & $26 \%$ \\
\hline Náusea & 5 & $16 \%$ \\
\hline
\end{tabular}

Observou-se que a dor de cabeça (60\%), a irritação (42\%) e a dificuldade de concentração (45\%) são os sintomas não auditivos mais comumente relatados pelos participantes e no que se refere aos aspectos de saúde auditiva, os participantes declaram apresentar cotidianamente sintomas de redução da audição (30\%) e de plenitude auricular (23\%). Vale destacar que estes sintomas podem ou não estar relacionados com à exposição ao ruído, uma vez que os participantes os declararam como sintomas constantemente presentes, não especificando o momento em que se manifestam.

Além disso, apenas 3 participantes $(10 \%)$ relataram sentir dificuldades de compreensão de fala nas situações sociais. Quando questionados sobre a percepção da audição em relação à lateralidade, $78 \%(n=24)$ dos participantes referem que há boa percepção auditiva em ambas as orelhas, $22 \%(n=7)$ refere que a orelha direita é melhor e nenhum participante relatou a orelha esquerda como melhor.

No que se refere aos efeitos auditivos e extra-auditivos decorrentes do uso de estéreos pessoais com fones de ouvido durante atividades físicas, a frequência dos sintomas pode ser vista detalhadamente na Tabela 3.

Tabela 3. Sintomas auditivos e extra auditivos apresentados pelos participantes após o uso de estéreos pessoais com fones de ouvido.

\begin{tabular}{c|c|c}
\hline Sintomas & Frequência Absoluta (n) & Frequência Relativa (\%) \\
\hline Plenitude Auricular & 12 & $40 \%$ \\
\hline $\begin{array}{c}\text { Redução da } \\
\text { audição }\end{array}$ & 9 & $30 \%$ \\
\hline Zumbido & 6 & $\mathbf{2 0 \%}$ \\
\hline Otalgia & 1 & $3 \%$ \\
\hline Dor de cabeça & 4 & $13 \%$ \\
\hline Insônia & 3 & $10 \%$ \\
\hline Vertigem & 1 & $3 \%$ \\
\hline $\begin{array}{c}\text { Alteração de } \\
\text { Pressão }\end{array}$ & 1 & $3 \%$
\end{tabular}

Tabela 4. Intensidade média do ruído ambiente das academias em dBNPS

\begin{tabular}{c|c|c|c|c}
\hline \multicolumn{5}{c}{ ACADEMIAS } \\
\hline $\begin{array}{c}\text { Intensidade } \\
\text { média do ruído } \\
\text { ambiente }\end{array}$ & Academia 1 & Academia 2 & Academia 3 & Academia 4 \\
\cline { 2 - 5 } & $78 \mathrm{~dB}$ & $81,4 \mathrm{~dB}$ & $77,1 \mathrm{~dB}$ & $77 \mathrm{~dB}$ \\
\hline
\end{tabular}

Tabela 5. Nível de pressão sonora dos estéreos pessoais dos participantes de acordo com o sexo e academia frequentada

\begin{tabular}{ccccc}
\hline Academia & NPS & $\begin{array}{c}\text { Sexo } \\
\text { Feminino }\end{array}$ & $\begin{array}{c}\text { Sexo } \\
\text { Masculino }\end{array}$ & Total \\
\hline \multirow{4}{*}{ Academia 1} & $70 \mathrm{~dB}-8 \mathrm{OdB}$ & 1 & 1 & 2 \\
& $81 \mathrm{~dB}-90 \mathrm{~dB}$ & 3 & 4 & 7 \\
& $91 \mathrm{~dB}-100 \mathrm{~dB}$ & 2 & 5 & 7 \\
Academia 2 & $101 \mathrm{~dB}-115 \mathrm{~dB}$ & 1 & 5 & 6 \\
& $91 \mathrm{~dB}-10 \mathrm{~dB}$ & 1 & 0 & 1 \\
Academia 3 & $101 \mathrm{~dB}-115 \mathrm{~dB}$ & 0 & 2 & 2 \\
& $81 \mathrm{~dB}-9 \mathrm{~dB} B$ & 1 & 1 & 2 \\
Academia 4 & $101 \mathrm{~dB}-100 \mathrm{~dB}-115 \mathrm{~dB}$ & 1 & 1 & 2 \\
\hline
\end{tabular}

A sensação de plenitude auricular aumentou de $23 \%$ relatados como presente no cotidiano para $40 \%$ dos participantes após o uso dos estéreos pessoais. Tal dado evidencia aumento da percepção do sintoma após utilização, ainda que o sintoma já estivesse presente em alguns dos participantes.

Da mesma forma, o zumbido, que foi relatado por $16 \%$ dos participantes como comum em seu cotidiano, passa a ser relatado por $20 \%$ dos participantes após o uso dos estéreos pessoais, dado semelhante ao de um estudo realizado com 85 sujeitos usuários de estéreos pessoais com fones de ouvido (mínimo de uma hora diária), no qual, estes responderam a um questionário sobre hábitos auditivos, o zumbido foi o sintoma auditivo relatado por $24,7 \%$ dos participantes ${ }^{16}$. Além disso, $30 \%$ dos participantes do presente estudo referem sentir redução da audição, contudo é necessário destacar que a mesma porcentagem havia referido este sintoma como presente no cotidiano.

Diante de tais dados, não é possível, portanto, afirmar que estes sintomas são decorrentes da exposição à música em intensidade elevada nos estéreos pessoais. Porém, da mesma forma, não é possível descartar essa relação, uma vez que os participantes possuem hábitos auditivos que 
podem gerar tais sintomas, amplamente discutidos na literatura sobre perdas auditivas causadas pela exposição aos níveis de pressão sonora elevados. O zumbido, por exemplo, é tido na literatura como uma das consequências do uso de estéreos pessoais ${ }^{17}$.

Autores como Luz e Borja ${ }^{18}$, afirmam que a sensação de plenitude auricular e o zumbido apresentam relação direta com o tempo de uso dos estéreos pessoais, pois quanto maior o tempo de exposição, maior a relação de sintomas auditivos. O estudo de Hanazumi et al. $^{16}$ com usuários de estéreos pessoais mostrou que aqueles que utilizam fones de ouvido por mais de cinco anos, apresentam maior risco de ter zumbido.

Outro estudo ${ }^{19}$ mostra que entre os sintomas mais comuns da perda auditiva induzida pelo ruído estão o zumbido, as dificuldades no entendimento da fala e a sensação de plenitude auricular. Cabe ressaltar que o zumbido pode causar interferência na concentração, no sono e na aprendizagem, tornando-se assim um sintoma importante a ser investigado, pois é comum nesta população ${ }^{20}$.

No que se refere aos efeitos extraauditivos, apesar da pequena porcentagem de sujeitos que referiram sintomas, os mais relatados pelos participantes foram a dor de cabeça (13\%) e a insônia (10\%), dado que corrobora com a literatura sobre o tema. Um estudo realizado com adultos usuários de estéreos pessoais, mostrou que $43 \%$ do grupo teve queixa de dor de cabeça ${ }^{15}$. Outro estudo realizado com 50 jovens que utilizavam dispositivos eletrônicos individuais, quando questionado os principais sintomas após o uso do dispositivo eletrônico, $44 \%$ dos jovens relataram a dor de cabeça ${ }^{7}$. Fiorini ${ }^{21}$, mostra que os efeitos à exposição ao som intenso podem gerar alterações extra-auditivas como 0 aumento da pressão sanguínea, interferência na comunicação oral, distúrbios do sono, incômodo, fadiga, dores de cabeça e irritabilidade.

Em relação à investigação da intensidade média do ruído ambiente das academias, houve variação entre 77 e 81,4 dBNPS (Tabela 4), reforçando o já encontrado na literatura da área. Um estudo em três academias, no qual foi realizada a medição do NPS das salas, encontrou resultados que ficaram entre 73,9 e 94,2 $\mathrm{dBA}^{22}$, valores próximos àqueles obtidos neste estudo.

De acordo com a NR-15, a exposição ao ruído de até $85 \mathrm{dBNPS}$ deve ser de no máximo oito horas diárias e a cada aumento de cinco dBNPS o tempo de exposição deve-se ser reduzido à metade ${ }^{23}$. Vale ressaltar que muitas academias chegam a funcionar por mais de oito horas diárias, expondo os profissionais que trabalham ao risco de alterações auditivas decorrentes da exposição ao ruído. Muitos professores de ginástica, durante suas aulas, utilizam o som com intensidade elevada, acreditando em um melhor rendimento para o grupo, mas esquecendo-se que pode oferecer risco aos alunos e a si próprio ${ }^{24}$. Mesmo que a música seja agradável para o grupo, a intensidade sonora elevada, pode proporcionar comprometimentos orgânicos, mentais e sociais $^{24}$.

Já no que se refere aos níveis de pressão sonora dos estéreos pessoais dos participantes, estes foram divididos por sexo e academia e podem ser vistos detalhadamente na Tabela 5. Constatou-se que a intensidade das músicas ouvidas pelos participantes em seus estéreos pessoais com fones de ouvido estavam, em média, 15 dBNPS acima do ruído ambiente da academia.

No presente estudo, 65\% ( $n=20)$ dos participantes utilizam estéreos pessoais entre 86 e 100dBNPS, mostrando-se alinhado a outros estudos realizado sobre o tema.

Um estudo realizado com 57 jovens, encontrou a intensidade dos estéreos pessoais entre o valor mínimo de $69 \mathrm{~dB}(\mathrm{~A})$ e o máximo de $93 \mathrm{~dB}(\mathrm{~A})^{25}$. Outro estudo realizado com 20 participantes, constatou que o valor médio da intensidade de seus estéreos pessoais é de $90,5 \mathrm{~dB}(\mathrm{~A})$, com valor máximo de $104 \mathrm{~dB}(\mathrm{~A})^{26}$.

Contudo, no presente estudo foram encontradas intensidades superiores a $100 \mathrm{dBNPS}$ nos equipamentos individuais, e conforme a NR-15 $5^{23}$, para a intensidade de 100 dBNPS, o tempo de exposição deve ser de, no máximo, uma hora. É possível inferir que a intensidade elevada da música nos estéreos pessoais dos participantes seja proveniente da necessidade de mascarar o ruído ambiente elevado da academia para ouvir apenas a música desejada de seu equipamento, visto que $39 \%(n=12)$ dos participantes relataram que a música ambiente da academia não os agrada.

Diante disso, infere-se que esses sujeitos estão expostos a um risco auditivo, visto que muitos relataram utilizar o dispositivo acima de tal intensidade por até duas horas, período em que estão na academia realizando suas atividades físicas. Outros autores observam que a PAIM pode estar se desenvolvendo para ser um problema social e de saúde pública ${ }^{27}$, pois os níveis de som dos fones de ouvido são altos e podem prejudicar a audição com algumas horas de uso ${ }^{28}$. 
Estar exposto a esse ruído pode trazer consequências, que irão ultrapassar o prejuízo para a audição e afetar a qualidade de vida dos sujeitos, pois há o risco de aparecimento de sintomas extra auditivos tais como a irritabilidade, o estresse, cefaleias constantes, perturbação do sono, doença cardiovascular e também podem prejudicar tarefas que exijam concentração, velocidade e a precisão de movimentos $^{29}$.

CONCLUSÃO

Com os dados obtidos no estudo é possível concluir que os efeitos auditivos mais relatados por este grupo de participantes após o uso de estéreos pessoais com fones de ouvido, foram a sensação de plenitude auricular, redução da audição e zumbido. Já os sintomas extra auditivos mais relatados foram a dor de cabeça e a insônia.

O NPS médio dos estéreos pessoais dos participantes foi de 95,4 dBNPS, variando de 70 a 115dBNPS. Sobre o ruído ambiente das academias, a intensidade média registrada foi de 78,1 dBNPS, variando de 77 e 81,4 dBNPS.

Sugere-se que para futuras pesquisas, sejam realizados estudos com uma quantidade maior de participantes e com maior variação de academias, para que se possa fazer um estudo comparativo entre indivíduos, sexo, intensidade de estéreo pessoal, ruído ambiente da academia e idade.

\section{REFERÊNCIAS}

1. Barcelos DD, Dazzi NS. Effects of the mp3 player on hearing. Rev CEFAC. 2014;16(3):779-91.

2. Andrade IFC, Russo ICP. Relationship between audiometric findings and auditory and extraauditory complaints of physical education teachers. Rev Soc Bras Fonoaudiol. 2010;15 (1):167-73.

3. Copeland BL, Franks BD. Effects of types and intensities of background music on treadmill endurance. J Sports Med Phys Fit. 1991;31: 100-3. apud. Santana, Luiz Diego Barreto. A influência da música na prática da musculação. Trabalho de Conclusão de Curso (graduação em Educação Física) - Universidade Estadual da Paraíba. 2014.

4. Szmedra L, Bacharach DW. Effect of music on perceived exertion, plasma lactate, norepinephrine and cardiovascular hemodynamics during treadmill running. Int $\mathrm{J}$ Sports Med. 1998;19(1):32-7.

5. Silva CL, Souza MF, Rossi Filho S, Silva LF, Rigon ACC. Leisure physical activity and health: a systematic review. Mudanças. 2017;25(1):57-65.
6. Simera MA. Avaliação audiológica, metabólica e de qualidade de vida no indivíduo jovem com zumbido usuário de estéreo pessoal [dissertação]. Marília: Faculdade de Filosofia e Ciências, UNESP; 2015.

7. Santana PDF, Mascarenhas WN, Borges LL, Camarano MRH. Hábitos de jovens usuários de dispositivos eletrônicos individuais e sintomas advindos da exposição à música em forte intensidade. EVS. 2015;42(3):315-26.

8. Swensson JRP, Swensson RP, Swensson RC. Ipod $(\mathrm{B}, \mathrm{mp} 3$ players and hearing. Rev Fac Ciênc Méd. 2009;11(2):4-5.

9. Leal SB, Silva SV, Momenshn-Santos TM, Fonseca ABM. Educational system to estimate the sound levels of earphones in teenagers. Distúrb Comum. 2018;30(4):759-66.

10. Mendes MH, Morata TC. Occupational exposure to music: a review. Rev Soc Bras Fonoaudiol. 2007;12(1):63-9.

11. Brasil. Ministério da Saúde. Ministério da Saúde, Secretaria de Atenção à Saúde, Departamento de Ações Estratégicas. Perda Auditiva induzida por ruído (Pair). Brasília: Ministério da Saúde, 2006. (Série A. Normas e Manuais Técnicos) (Saúde do trabalhador; 5 Protocolos de complexidade diferenciada).

12. Araújo MF, Navarro F. Supplement consumption among fitness center user in Linhares, Espírito Santo, Brazil. Rev Bras Nutr Esp. 2008;2(8):46-54.

13. Silva JR, Gress FAG. The influence of music and musical rhythms in exercise endurance. Rev Acta Bras do Mov Hum. 2012;2(4):46-59.

14. Moura NL, Grillo DE, Merida M, Campanelli JR, Merida $F$. The relationship between music and motivation among women who practices gyminastic at fitness clubs. Rev Mackenzie Educ Fís Esp. 2007;6(3):103-18.

15. Gonçalves CL, Dias FAM. Audiological findings in young users of headphones. Rev CEFAC. 2014;16(4):1097-108.

16. Hanazumi A, Gil D, lório MCM. Hearing habits and audiological evaluation in adults. Audiol Commun Res. 2013;18(3):179-85.

17. Park B, Choi HG, Lee HJ, An SY, Kim SW, Lee JS et al. Analysis of the prevalence of and risk factors for tinnitus in a young population. Otol Neurotol. 2014;35(7):1218-22.

18.da Luz TS, Borja AL. Hearing symptoms personal stereos. Int Arch Otorhinolaryngol. 2012;16(2):163-69.

19.Dias A, Cordeiro R, Corrente JE, Gonçalves CGO. Association between noise-induced hearing loss and tinnitus. Cad Saúde Pública. 2006;22(1): 63-8.

20. Coelho CB, Sanchez TG, Tyler RS. Tinnitus in children and associated risk factors. Prog Brain Res. 2007;166:179-91. 
21. Fiorini AC. Efeitos Não Auditivos Do Ruído. In: Boéchat EM, Menezes PL, Couto CM, Frizzo ACF, Scharlach RC, Anastasio ART (org). Tratado de Audiologia. Capitulo 26. 2. ed. Rio de Janeiro: Guanabara Koogan; 2015.

22. Lacerda ABM, Morata TC, Fiorini AC. Characterization of sound pressure levels in fitness centers and complaints from instructors. Rev Bras Otorrinolaringol. 2001;67(5):656-59.

23. Norma Regulamentadora 15. Atividades e operações insalubres, CLT Capítulo V, Título II. Diário Oficial da União 1978; 8 jun.

24. Oliveira GC, Silva CC. Nível de ruído nas aulas de ginástica e as queixas auditivas apresentadas pelos professores. Hórus. 2010;5(2):276-83.

25. Silva ED, Teixeira JAMS, Scharlach RC. Use of digital audio players by high school students: measurement of use intensity and usage habits. CoDAS. 2018;30(5):1-9.

26. Côrtes-Andrade IF, Souza AS, Frota SMMC. Distortion product evoked otoacoustic emissions study with individuals of a fitness gym. Rev CEFAC. 2009;11(4):654-61.

27. Vogel I, Brug J, Hosli EJ, Van Der Ploeg CPB, Raat H. MP3 players and hearing loss: adolescents perceptions of loud music and hearing conservation. J Pediatr. 2008;152(3): 400-4.

28. Kim MG, Hong SM, Shim HJ, Kim YD, Cha Cl, Yeo SG. Hearing threshold of korean adolescents associated with the use of personal music players. Yonsei Med J. 2009;50(6): 771-76.

29. Cavalcanti TLO, Andrade WTL. Auditory and extra-auditory effects of noise on dentist's health. R Bras Ci Saúde. 2012;16(2):161-66.

\section{CONFLITO DE INTERESSES}

Os autores declaram não haver conflitos de interesse

AUTOR PARA CORRESPONDÊNCIA

\section{Juliana De Conto}

PR 153, Km 7, s/n - Riozinho 84500-00 Irati - PR, Brasil

Email: jdconto@yahoo.com.br
Submetido em 30/03/2020

Aceito em 23/10/2020 